\title{
Brain and odor: I. Alteration of human EEG by odor administration
}

\author{
TYLER S. LORIG and GARY E. SCHWARTZ \\ Yale University, New Haven, Connecticut
}

\begin{abstract}
Two experiments examined the relationship between time domain patterns of EEG activity and self-reports for individuals exposed to different odorants. In the first experiment, spiced apple, eucalyptus, and lavender odors were evaluated. The three odorants produced consistently different patterns of EEG theta activity and self-reports. These results suggest that odor administration is a reliable variable in manipulating neurophysiological response systems and may be influential in affecting human performance and mood. In order to assess the sensitivity of EEG in the delineation of odor effects, a second experiment was conducted. EEG activity was recorded while subjects smelled five similar commercial odorous chemicals and an unscented base. Subjects completed questionnaires on odor character and mood following each presentation. Results of the experiment indicated little perceptual or mood difference produced by the odors. EEG alpha and theta activity in the left and right hemispheres, however, differed widely depending upon the odor presented and was dissociated from self-report. These results suggest the sensitivity of EEG techniques in odor experiments and indicate that the perceptual component of olfaction accounts for only a limited part of the central nervous system changes produced by smelling an odor.
\end{abstract}

Anecdotal literature is filled with accounts of odors being used to alter mood, alertness, and sexual arousal (Billot \& Wells, 1975; Morris, 1984; Valnet, 1982). Although these supposed odor effects should be approached with skepticism, the close association between the olfactory and limbic systems provides a means by which many of these effects might be realized. The limbic system is, for instance, known to be associated with mood alteration, memory, and sexual activity (Isaacson, 1974). Olfactory input is provided to a variety of telencephalic structures within the central nervous system (CNS) (see Castellucci, 1985, for review). It follows from the architecture of this system that olfactory stimuli should produce widespread activity across the forebrain and that some of this activity may be represented in the EEG even though much of the olfactory system is subcortical. Systematic studies of odor effects on humans that examine limbic activity, such as EEG theta activity, are rare.

In recent studies, Schwartz, Whitehorn, Hernon, and Jones (1986a, 1986b) found that odors altered cardiovascular and respiratory patterns and self-report differences, indicating that a particular odor, spiced apple

This research was supported by a gift to Yale University by International Flavors and Fragrances, Inc. (G. E. Schwartz, principal investigator). The authors wish to thank A. Wagner, D. Kraemer, W. Cain, C. Warren, S. Warrenburg, M. Monteneau, the editor, and two anonymous reviewers for their comments and review of a preliminary draft of this manuscript. C. Crandal and J. Cacioppo provided statistical advice. The authors also wish to thank K. Herman, H. Cameron, and P. Walker for their assistance in collection and analysis of the data. T. S. Lorig's current address is Department of Psychology, Washington and Lee University, Lexington, VA 24450 . G. E. Schwartz's current address is Department of Psychology, University of Arizona, Tucson, AZ 85721 . (available from International Flavors and Fragrances, Union Beach, NJ), was stress reducing and relaxing yet did not produce drowsiness. Relaxation effects have been associated with EEG patterns of increased alpha activity that are especially prevalent across posterior areas of the head (Lehrer, Woolfolk, Rooney, McCann, \& Carrington, 1983; Ray \& Cole, 1985).

The experiments described here used EEG period analysis to assess the effects of odor administration. Period analysis is a time domain technique that has proven useful in a variety of cognitive and drug studies (Fink, 1975). The technique quantifies the number of waves that occur in various EEG frequencies rather than their amplitude, which is the case with spectral analysis. Comparisons of period and spectral analyzed data indicate that period analysis has greater sensitivity during task performance (Lorig, 1986), and therefore would seem better suited to studies of EEG associated with olfactory phenomena.

\section{EXPERIMENT 1}

\section{Method}

Subjects. Nine subjects ( 4 males and 5 females) who responded to a posted advertisement participated in the experiment. All subjects were right-handed and were screened for familial history of left-handedness, head injuries, and drug use. They were between the ages of 18 and 24 and were paid for their participation.

Materials and Procedure. The subjects entered the laboratory and were told the nature of the experiment and the procedures for collection of EEG data. After informed consent was obtained, 10mm cup-type gold-plated electrodes were affixed at four locations (F7, T5, F8, and T6, referenced to linked mastoids) using Grass electrode cream. Electrode impedance was always below $10 \mathrm{k} \Omega$ and was typically less than $5 \mathrm{k \Omega}$. EEG was amplified and filtered to a 1 - to $1000-\mathrm{Hz}$ bandwidth using Coulbourn Instruments biotelem- 
etry amplifiers interfaced to an IBM PC compatible data-acquisition system (12-bit resolution, $128-\mathrm{Hz}$ sampling rate). EEG data were digitally smoothed off-line to a 2- to $32-\mathrm{Hz}$ bandpass $(-3 \mathrm{~dB})$. Artifacts, such as subjects' postural movements and eye movements of less than $4 \mathrm{~Hz}$, were excluded from data analysis by the period analysis algorithm. Artifacts typically constituted less than $5 \%$ of subject records.

The subjects smelled the contents of four 5-ml vials, presented in counterbalanced order. The interstimulus interval was approximately $4 \mathrm{~min}$. Three vials contained the odors spiced apple, eucalyptus, and lavender prepared in a diethylphthalate (DEP) base. Lavender and eucalyptus are essential oils and were diluted to $60 \%$ in DEP. The fourth vial contained only distilled water. The odors were presented $5 \mathrm{~cm}$ from the subjects' noses for $1 \mathrm{~min}$ each. The room was ventilated during the $3 \mathrm{~min}$ between odor presentations at the experimenters' discretion. The subjects were instructed to sit quietly with eyes closed and to breathe through their noses during the odor trials.

The odor trials were embedded in a series of other odor and mental tasks, such as mental arithmetic and imagery. A resting baseline condition was also included in this experiment. The subjects were asked to complete a 17-item questionnaire assessing patuerns of emotion between the odor and imagery/arithmetic trials. The questionnaire consisted of 10-point Likert scales associated with various terms such as "relaxed," "tense," "happy," and "bored." It also contained questions relating to the pleasantness of and memories associated with the odors.

\section{Results and Discussion}

Raw EEG data were period analyzed (Lorig, 1986), and the amounts of activity in the alpha $(8-13 \mathrm{~Hz})$ and theta (4-7 Hz) ranges were extracted for each of three 10-sec epochs that occurred at the beginning, middle, and end of the 1-min trial. Because the sample size in this experiment fell below the criterion stated by Vasey and Thayer (1987) $(N=k+20)$, Greenhouse-Geisser adjusted repeated measures analyses of variance were used to provide adequate protection against sphericity.

Results of the analysis of variance for theta activity (odor $x$ epoch $x$ hemisphere $x$ anterior/posterior) indicated a significant difference in the amount of EEG theta activity produced by smelling the different odorants compared with smelling the no-fragrance control $[F(3,24)=$ 5.41, Greenhouse-Geisser corrected $p=.013$ ] (see Figure 1). These findings replicated extensive pilot testing of theta activity associated with these odors. Contrasts of the various odor conditions using repeated measures analysis of variance indicated that the spiced apple odorant differed from the lavender odorant $[F(1,8)=29.97$, $p=.0006]$ and tended to differ from the eucalyptus odorant $[F(1,8)=4.25, p=.073]$.

Although analysis of alpha activity did not indicate a significant odorant effect, the no-odor control condition seemed to produce less alpha activity than the other odors. This was also the case in pilot testing and appeared to be related to the fact that subjects expected to smell some odor. This suggests that this type of condition is an inappropriate control condition for experiments of this nature.

Although differences in EEG theta activity may be due to differences in the intensity of the odors, one-way repeated measures analysis of variance of the questionnaire data associated with each of these odors indicated no differences in their perceived intensity or pleasantness. Further analysis of these rating scale data (Michell, 1986) revealed that only 2 of the 15 questionnaire items indicated differences in self-reports for the odors. These analyses indicated that spiced apple and eucalyptus odorants were associated with reports of significantly less anxiety $[F(2,16)=3.77, p=.045]$ and less tension $[F(2,16)=$
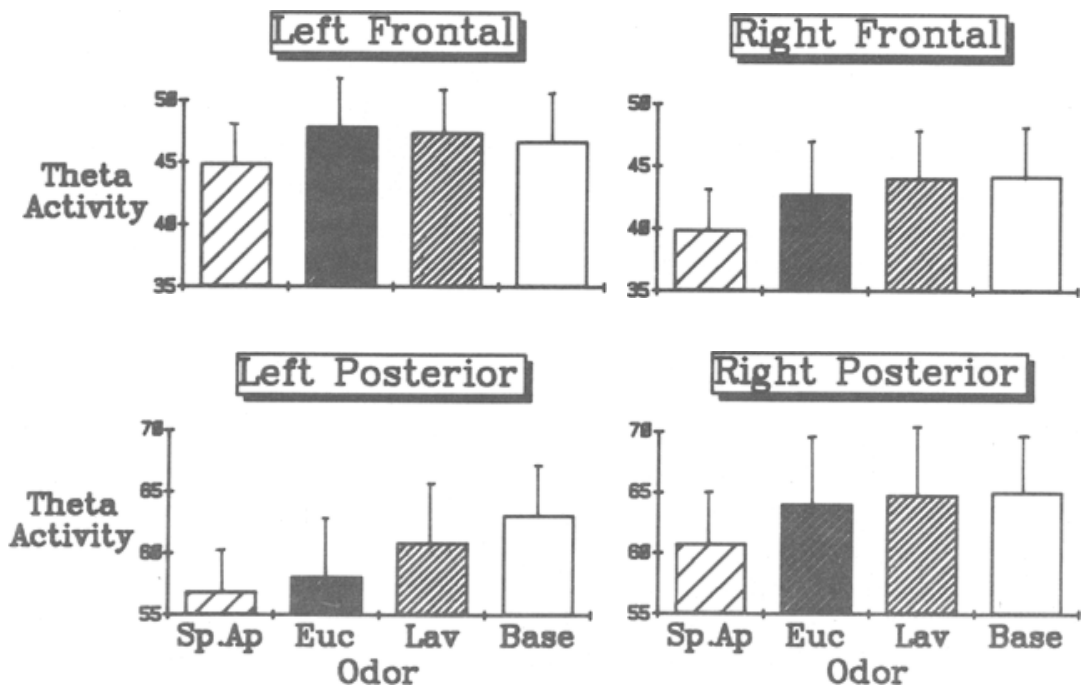

Figure 1. Mean amount of theta activity for each odorant (spiced apple, eucalyptus, and lavender) at each electrode location. Error bars indicate standard errors of the means. 
$3.62, p=.05]$ than was the lavender odorant. Theta activity differences have been associated with emotion- and anxiety-provoking stimuli (Ahern \& Schwartz, 1985), and may account for the self-report differences found with lavender odorant.

These data indicate the salience of odors as neurophysiological stimuli, even in cases in which subjects do not report hedonic or intensity differences between odors. Because of this salience and the ability to modify limbic system activity, odors may have application in the alteration of mood states in humans. The findings of this experiment also indicate that odors may continue to exert CNS effects even after initial exposure and suggest that ambient odor effects may be found.

\section{EXPERIMENT 2}

Experiment 1 demonstrated that different odors produced EEG alterations that were related to subjects' self-reports. The odors used in Experiment 1 differed in odor quality and were rated by subjects as moderate to strong in intensity. Because of the differences in the nature of the odors presented in that experiment, it is not clear whether the EEG differences found represented fundamental changes in CNS activity induced by the odorants, or changes produced by cognitive/memorial activity associated with the odorants.

In another experiment on this topic (Lorig \& Schwartz, 1987), EEG was examined while subjects imagined food odors. The results of this experiment suggested that the decreased theta activity found when subjects smelled a spiced apple odorant (first experiment) may have been due to the odor's association with food stimuli, since images of food were also found to reduce theta activity. Although these findings offer some clarification of effects found with the spiced apple odorant, they do not answer the more fundamental question about the nature of neural responses to different odors or the ability of EEG techniques to measure such responses.

To assess the effects of different odors while minimizing their memorial and cognitive differences, it is necessary to use perceptually similar odorants. For this reason, refined fine fragrances of similar odor qualities were used in the present study.

\section{Method}

Subjects. Ten subjects ( 4 males and 6 females) participated. The subjects were right-handed and ranged in age from 18 to 56 , with a mean age of approximately 24 . They reported no history of head injuries and were not taking any medication known to alter EEG. All subjects were paid for their participation.

Materials and Procedure. Materials used in this study were identical to those used in Experiment 1, except the fragrances differed. Participants were seated comfortably with eyes closed while EEG data were collected. Five fine-fragrance odorant trials were presented at the end of a series of cognitive and other odor tasks. The fine fragrances used in the study, provided by International Flavors and Fragrances (Union Beach, NJ), consisted of five floral note perfumes prepared in an unscented detergent base (L1-L5). One trial consisted of the detergent base only (base). All six samples were diluted to a $5 \%$ solution in distilled water. The odorant samples were presented to the subjects in $5-\mathrm{ml}$ vials placed $5 \mathrm{~cm}$ from their noses. Data were collected in three 10-sec epochs at the beginning, middle, and end of a 1-min period for each fragrance. The order of presentation of the fragrances was counterbalanced. Questionnaires concerned with odor character and mood were administered at the end of each trial. Following completion of the experiment, the subjects were debriefed and paid.

\section{Results and Discussion}

Period analysis of the EEG reduced the data to a histogram of the number of waves of various frequencies that occurred during each of the three 10 -sec data collection epochs for each task. The number of waves corresponding to the alpha $(8-13 \mathrm{~Hz})$ and theta $(4-7 \mathrm{~Hz})$ frequency ranges were extracted and the five odorants compared using a repeated measures analysis of variance (odor $x$ epoch $\times$ hemisphere $x$ anterior/posterior), corrected for sphericity. The baseline condition was not included in the analysis because it represented a trial in which odor information was markedly different from trials with the other fragrances and thus would have artificially biased the omnibus $F$ test. The difficulties in establishing a representative control condition in odor experiments of this type are discussed in Experiment 1.

Results of the repeated measures analysis indicated that alpha activity was marginally greater in posteriorly placed electrodes $[F(1,9)=4.93, p=.053]$ and increased in those locations over time $[F(2,18)=6.30$, GreenhouseGeisser adjusted $p=.013$, as would be expected from previous research (Adrian \& Mathews, 1934; Lorig, 1986). The five odor conditions were also found to produce differences in the amount of alpha activity over the left and right hemispheres, which differed over time $[F(8,72)=3.04$, Greenhouse-Geisser adjusted $p=.032]$.

Analysis of theta activity indicated similar differences. The five odors produced differing patterns of hemispheric activity over the three epochs of data collection $[F(8,32)$ $=2.84$, Greenhouse-Geisser adjusted $p=.040$; see Figure 2]. As is evident in Figure 2, most of the odorants produced a pattern of greater left hemisphere theta activity during epoch 1 . Hemispheric activity tended to become more equal for all odorants except $L A$ during epoch 2 . In epoch $3, L 4$ reversed its pattern and L2 became more assymetrical. This finding is interesting since subjective responses to $L 2$ and $L 4$ fragrances were very similar.

Repeated measures analyses of the self-report data (Michell, 1986) obtained following exposure to each odor indicated that of the 15 items examined, only perceived intensity differed among the odors $[F(4,32)=5.28$, $p=.002$ ]. L2 was judged to have the greatest intensity, and L5 was reported least intense. It is unlikely that the self-report scale used was insensitive to the experimental manipulations since our previous research with this scale has produced reliable differences.

The results of this and the previous experiment indicate that odors affect CNS activity and, in particular, EEG 


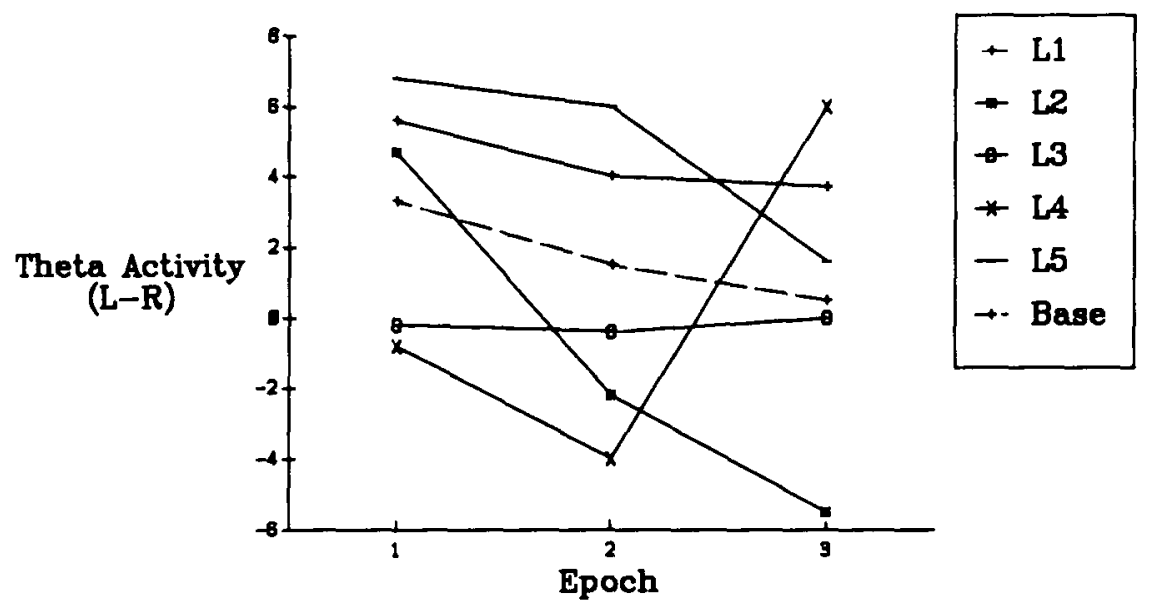

Figure 2. Hemisphere distribution (left electrode pair minus right electrode pair) of theta activity across three epochs for each odorant.

theta activity. This alteration of activity may mediate changes in subjective mood states. Findings from the present investigation, however, indicate that even odors of high perceptual similarity can produce very different patterns of neurophysiological activity. These findings suggest that perceptual phenomena associated with odors are only a small part of the overall picture of CNS activity that accompanies the inhalation of odorous air.

\section{REFERENCES}

Adrian, E. D., Mathews, B. H. C. (1934). The Berger thythm: Potential changes from the occipital lobe of man. Brain, 57, 355-385. Ahern, G., SCHWARTz, G. (1985). Differential lateralization for positive and negative emotion in the human brain: EEG spectral analysis. Neuropsychologia, 23, 745-755.

Billot, M., * Wells, F. (1975). Perfumery technology: Art, science, industry. New York: Wiley.

Castellucci, V. F. (1985). The chemical senses: Taste and smell. In E. R. Kandel \& J. H. Schwartz (Eds.), Principles of neural science (2nd ed., pp. 409-428). New York: Elsevier.

FinK, M. (1975). Cerebral electrometry-quantitative EEG applied to human psychopharmacology. In G. Dolce \& H. Kunkel (Eds.), CEAN-Computerized EEG analysis (pp. 271-288). Stuttgart, West Germany: Fischer.

IsAacson, R. L. (1974). The limbic system (2nd ed.). New York: Plenum Press.
Lehrer, P. M., Woolfolk, R. L., Rooney, A. J., MCCANN, B., \& Carrungton, P. (1983). Progressive relaxation and meditation: A study of the psychophysiological and therapeutic differences between two techniques. Behavior Research Therapy, 21, 651-662.

LoRu, T. S. (1986). EEG and task performance: A comparison of three analytic techniques. Physiological Psychology, 14, 130-132.

Lorig, T. S., SCHWARTZ, G. E. (1987). EEG activity during food and relaxation imagery. Psychophysiology, 24, 599.

MiCHELL, J. (1986). Measurement scales and statistics: A clash of paradigms. Psychological Bulletin, 100, 398-407.

MoRrIs, E. T. (1984). Fragrance. New York: Scribners.

RAY, W. J., COLE, H. W. (1985). EEG alpha activity reflects attentional demands and beta reflects emotional and cognitive processes. Science, 228, 750-751.

Schwartz, G. E., Whitehorn, D., Hernon, J. C., \& Jones, M. (1986a). The ARC method for averaging repetitive cycles: Application to respiration during stress and relaxation. Psychophysiology, 23, 460 .

Schwartz, G. E., Whitehorn, D., Hernon, J. C., Jones, M. (1986b). Subjective and respiratory differences of fragrances: Interaction with hedonics. Psychophysiology, 23, 460.

VALNET, J. (1982). The practice of aromatherapy. Essex, England: Daniel.

VASEY, M. W., \& ThaYeR, J. F. (1987). The continuing problem of false-positives in repeated measures ANOVAs in psychophysiology. Psychophysiology, 24, 479-486.

(Manuscript received March 14, 1988; revision accepted for publication June 15, 1988.) 